\title{
A Sequent Calculus for a Negative Free Logic
}

\section{Abstract}

This article presents a sequent calculus for a negative free logic with identity, called $\mathbf{N}$. The main theorem (in part 1) is the admissibility of the Cut-rule. The second part of this essay is devoted to proofs of soundness, compactness and completeness of $\mathbf{N}$ relative to a standard semantics for negative free logic.

Keywords: Free Logic, Cut-elimination, Compactness, Completeness, Existence

\section{INTRODUCTION $^{1}$}

K. Lambert introduced the term 'free logic' in the late 1960s. A standard definition of free logic is the following: A logic $S$ is a free logic iff (1) $S$ is free of existential presuppositions with respect to the singular terms of $S$, (2) $S$ is free of existential presuppositions with respect to the general terms of $S$ and finally (3) the quantifiers of $S$ have existential import. There are three "families" of free logics: positive, negative and neutral. For our purposes it suffices to define a negative free logic: A negative free logic is a free logic, where each simple statement containing at least one empty singular term is false. E.g. 'Vulcan is (identical with) Vulcan' is false; and so is 'Vulcan rotates' (Cf. Lambert (1997, 81ff)). It should be mentioned that there is no particular formal system called "the" negative free logic, but there is a whole family of such systems. Since the sentence "Vulcan is (identical with) Vulcan' is false in negative free logics, a general feature of these is that identity statements can only be true just in case they contain no non-denoting singular term (cf. Hintikka (1964), Schock (1969)). So, negative free logics have a non-standard identity theory, unlike positive free logics. Scales (1969) in his pioneering work in negative free logic presents strong arguments in favor of this family of free logics in general.

The history of negative free logic can be traced back to Aristotle; a clear example for this is the following passage: "It might, indeed, very well seem that the same sort of thing does occur in the case of contraries said with combination, 'Socrates is well' being contrary to 'Socrates is sick'. Yet not even with these is it necessary always for one to be true and the other false. For if Socrates exists one will be true and one false, but if he does not both will be false; neither 'Socrates is sick' nor 'Socrates is well' will be true if Socrates himself does not exist at all."'

\footnotetext{
${ }^{1}$ Research on this paper was funded by the FWF (P17392-G04).

${ }^{2}$ Aristotle $(1984,21)$
} 
Scott (1967) argued explicitly that free logic is relevant in some fields of mathematics and computer science. Though, as Gumb $(2000,2001)$ stresses the term 'free logic' is not in use in the literature of mathematics and computer science.

G. Gentzen (1934/35) introduced sequent calculi as a new kind of formal system. These are not as close to actual reasoning as natural deduction systems, but they have very interesting metalogical properties. The most famous one is the Hauptsatz or the normal form theorem. In this essay we introduce a sequent calculus for negative free logic with identity and prove the Hauptsatz for this system. Consistency and the theorem concerning the sub-formula property are easily provable, given the proof of the Hauptsatz. The sub-formula property is one salient feature of sequent calculi because it follows from it that the information required for a derivation of a sequent is contained in the sequent itself. For this reason sequent calculi are particularly interesting for automated theorem proving. In contrast to this kind of formal system Hilbert-style formal systems lack the subformula property. This is so, because in general modus ponens deprives formulas of the necessary information for their derivation. So far there is no sequent calculus fpr negative free logic in the literature on free logic. In section 1 a corresponding sequent calculus - called $\mathbf{N}$ - is introduced. In the second part soundness, compactness and completeness is proved for the system in question relative to a standard semantics for negative free logics. The completeness theorem provides us with another proof of the Hauptsatz but it is not constructive, i.e. this proof does not give us a procedure for effectively eliminating cuts.

Before we present the formal system we have to state the language and some definitions.

\section{Language $L$}

The alphabet of $L$ consists of

(i) a denumerably infinite set of free individual variables (FV)

(ii) a denumerably infinite set of bound individual variables

(iii) an at most denumerably infinite set of (for any $n$ ) $n$-ary predicate symbols

(iv) $\neg, \rightarrow, \Rightarrow, \forall, \mathrm{E}$ !, $=$

We use as syntactical variables (with and without indices):

$a, b \quad$ for free individual variables

$x, y \quad$ for bound individual variables

$s, t \quad$ for terms 
$F^{n}, G^{n} \quad$ for $n$-ary predicate symbols

$\mathbf{D}, \mathbf{G} \quad$ for prime formulas

A, B, C, .. for formulas

$\Gamma, \Delta, \Psi, \Phi$ are finite (possibly empty) sequences of formulas

Terms, formulas and sequents

(1) Every free individual variable is a term.

(2) If $t_{1}, \ldots, t_{\mathrm{n}}$ are terms and $F^{n}$ is an n-ary predicate, then $F^{n} t_{1} \ldots t_{\mathrm{n}}$ is a formula.

(3) If $t$ is a term, then $\mathrm{E} ! t$ is a formula.

(4) If $s$ and $t$ are terms, then $s=t$ is a formula.

(5) If $\mathrm{A}$ and $\mathrm{B}$ are formulas, then $\neg \mathrm{A}, \mathrm{A} \rightarrow \mathrm{B}$ are formulas.

(6) If $\mathrm{A}[a]$ is a formula, s.t. in $\mathrm{A}$ the bound variable $x$ does not occur, then $\forall x \mathrm{~A}[x]$ is a formula.

(7) If $A_{1}, \ldots, A_{n}$ and $B_{1}, \ldots, B_{m}$ are formulas, then $A_{1}, \ldots, A_{n} \Rightarrow B_{1}, \ldots, B_{m}$ is a sequent. (The left part of $A_{1}, \ldots, A_{n} \Rightarrow B_{1}, \ldots, B_{m}$ is called the antecedent and the right part succedent. Intuitively, the antecendent is interpreted as a conjunction of the formulas occurring in the antecedent, i.e. $A_{1} \wedge \ldots \wedge A_{n}$; and the succedent is interpreted as a disjunction of the formulas occurring in the succedent, i.e. $B_{1} \vee \ldots \vee B_{m}$ )

The notation $\mathrm{A}[a]$ is explained in the following way: the free variable $a$ occurs in A in several distinguished places. $\mathrm{A}[x]$ is the formula which is obtained from $\mathrm{A}[a]$ by substituting the bound variable $x$ for the distinguished occurrences of the free variable $a$ in $\mathrm{A}[a]$.

$\wedge, \vee, \leftrightarrow, \exists$ are defined as usual. Prime formulas are all formulas which are constructible by the clauses (2) - (4).

For the proof of the Hauptsatz it is important to have the notion of the 'degree of a formula':

Inductive definition of the degree of a formula

(G1) $g(\mathrm{~A})=0$, if A is prime.

$(\mathrm{G} 2) g(\mathrm{~A} \rightarrow \mathrm{B})=g(\mathrm{~A})+g(\mathrm{~B})+1$

(G3) $g(\neg \mathrm{A})=g(\mathrm{~A})+1$

(G4) $g(\forall x \mathrm{~A}[x])=g(\mathrm{~A})+1$ 


\section{THE FORMAL SYSTEM N}

\section{Axioms}

(Ax1) $\mathbf{D}, \Gamma \Rightarrow \Delta, \mathbf{D}$

(Ax2) $\mathrm{E} ! t, \Gamma \Rightarrow \Delta, t=t$

(Ax3) $s=t, \mathbf{D}[s], \Gamma \Rightarrow \Delta, \mathbf{D}[t]$

(Ax4) $\mathbf{D}[t], \Gamma \Rightarrow \Delta, \mathrm{E} ! t$

\section{Rules}

Structural rules

$$
\begin{gathered}
(\mathrm{P} \Rightarrow) \frac{\Gamma, \mathrm{A}, \mathrm{B}, \Delta \Rightarrow \Psi}{\Gamma, \mathrm{B}, \mathrm{A}, \Delta \Rightarrow \Psi} \\
\text { Logical rules }
\end{gathered}
$$

\section{Logical rules}

$$
(\Rightarrow \mathrm{P}) \frac{\Gamma \Rightarrow \Delta, \mathrm{A}, \mathrm{B}, \Psi}{\Gamma \Rightarrow \Delta, \mathrm{B}, \mathrm{A}, \Psi}
$$

$$
(\neg \Rightarrow) \frac{\Gamma \Rightarrow \Delta, \mathrm{A}}{\neg \mathrm{A}, \Gamma \Rightarrow \Delta}
$$

$(\rightarrow \Rightarrow) \frac{\Gamma \Rightarrow \Delta, \mathrm{A} \quad \mathrm{B}, \Gamma \Rightarrow \Delta}{\mathrm{A} \rightarrow \mathrm{B}, \Gamma \Rightarrow \Delta}$

$(\forall \Rightarrow) \underline{\mathrm{A}[t], \forall x \mathrm{~A}[x], \Gamma \Rightarrow \Delta \quad \forall x \mathrm{~A}[x], \Gamma \Rightarrow \Delta, \mathrm{E} ! t}$

$$
\forall x \mathrm{~A}[x], \Gamma \Rightarrow \Delta
$$

$(\Rightarrow \forall) \underline{E} \underline{E}, \Gamma \Rightarrow \Delta, \mathrm{A}[a]$

$$
\Gamma \Rightarrow \Delta, \forall x \mathrm{~A}[x]
$$

(I-Cut)

$$
\frac{\Gamma \Rightarrow \Delta, s=t \quad s=t, \Gamma \Rightarrow \Delta}{\Gamma \Rightarrow \Delta}
$$

\section{Remarks}

- (V!) means that the free variable $a$ must not occur below the inference line.

- One distinctive feature of free logic is that although it allows for its singular terms to be empty, the quantifiers of free logic retain their existential import. That 
the quantifiers have existential import in this logic is seen by the rules $(\forall \Rightarrow)$ and $(\Rightarrow \forall)$.

- The last rule - (I-Cut) - termed inessential cut by Takeuti (Takeuti (1987, p. 40). It is 'inessential' because the only cut-formulas admitted by (I-Cut) are equality formulas.

Inductive definition of derivability in $\mathbf{N}$ :

(i) Every axiom is derivable in $\mathbf{N}$.

(ii) If the premise(s) of a (basic) rule of inference is (are) derivable in $\mathbf{N}$, so is the conclusion of this (basic) inference derivable in $\mathbf{N}$.

A formula A is provable in $\mathbf{N}$ iff the sequent $\Rightarrow \mathrm{A}$ is derivable in $\mathbf{N}(\vdash \mathrm{A})$.

An application of a logical rule is a logical inference. If $\Gamma \Rightarrow \Delta$ is a derivable sequent in $\mathbf{N}$, then $h(\Gamma \Rightarrow \Delta)$ denotes the minimal number of logical inferences that are necessary for a derivation of $\Gamma \Rightarrow \Delta$. We call $h(\Gamma \Rightarrow \Delta)$ the height of the sequent $\Gamma \Rightarrow \Delta$.

\section{Definitions}

A rule is called admissible in $\mathbf{N}$ provided that if the premises of the rule are derivable in $\mathbf{N}$ then the conclusion of this rule is derivable in $\mathbf{N}$.

A rule is called directly admissible in $\mathbf{N}$ if it is admissible and each application of the rule can be replaced by a finite number of basic rules.

A rule is called indirectly admissible in $\mathbf{N}$ if it is admissible but not directly admissible.

The most important difference between directly and indirectly admissible rules is that indirectly admissible rules are not invariant to extensions of the system. ${ }^{3}$

\subsection{Some directly admissible rules}

$$
\begin{array}{ll}
(\vee \Rightarrow) \frac{\mathrm{A}, \Gamma \Rightarrow \Delta \quad \mathrm{B}, \Gamma \Rightarrow \Delta}{\mathrm{A} \vee \mathrm{B}, \Gamma \Rightarrow \Delta} & (\Rightarrow v) \frac{\Gamma \Rightarrow \Delta, \mathrm{A}, \mathrm{B}}{\Gamma \Rightarrow \Delta, \mathrm{A} \vee \mathrm{B}} \\
(\wedge \Rightarrow) \frac{\mathrm{A}, \mathrm{B}, \Gamma \Rightarrow \Delta}{\mathrm{A} \wedge \mathrm{B}, \Gamma \Rightarrow \Delta} & (\Rightarrow \wedge) \frac{\Gamma \Rightarrow \Delta, \mathrm{A} \quad \Gamma \Rightarrow \Delta, \mathrm{B}}{\Gamma \Rightarrow \Delta, \mathrm{A} \wedge \mathrm{B}}
\end{array}
$$

$(\exists \Rightarrow) \mathrm{E} ! a, \mathrm{~A}[a], \Gamma \Rightarrow \Delta(\mathrm{V} !)$

\footnotetext{
${ }^{3}$ For further details (and examples) on directly/indirectly admissible rules see Schütte (1960, pp. 44f.).
} 


$$
\exists x \mathrm{~A}[x], \Gamma \Rightarrow \Delta
$$

$(\Rightarrow \exists) \underline{\Gamma \Rightarrow \Delta, \exists x \mathrm{~A}[x], \mathrm{A}[t] \quad \Gamma \Rightarrow \Delta, \exists x \mathrm{~A}[x], \mathrm{E} ! t}$

$$
\Gamma \Rightarrow \Delta, \exists x \mathrm{~A}[x]
$$

All these rules are easily derivable by the definitions of the connectives and of the existential quantifier.

\subsection{Some indirectly admissible rules}

\subsubsection{Substitution (Sub)}

$\Gamma[a] \Rightarrow \Delta[a]$

Where: $a$ does not occur under the

$\Gamma[b] \Rightarrow \Delta[b]$ inference line

is admissible in $\mathbf{N}$ and it holds that $h(\Gamma[b] \Rightarrow \Delta[b]) \leq h(\Gamma[a] \Rightarrow \Delta[a])$

\section{Proof}

There is a derivation of $\Gamma[a] \Rightarrow \Delta[a]$ in $\mathbf{N}$ in tree-form (see e.g. Gentzen 1934/35). If therein occurs a $(\Rightarrow \forall)$-inference, s.t. the variable $b$ is subject to the condition on variables, then this variable will be replaced by a new variable which does not occur in the premises of this inference and which does not occur in any sequents that occur above this premise. Hence, a derivation of $\Gamma[b] \Rightarrow \Delta[b]$, where $h(\Gamma[b]$ $\Rightarrow \Delta[b]) \leq h(\Gamma[a] \Rightarrow \Delta[a])$ is the case, is obtainable.

\subsubsection{Weakening}

$$
(\mathrm{W} \Rightarrow) \frac{\Gamma \Rightarrow \Delta}{\mathrm{A}, \Gamma \Rightarrow \Delta} \quad(\Rightarrow \mathrm{W}) \frac{\Gamma \Rightarrow \Delta}{\Gamma \Rightarrow \Delta, \mathrm{A}}
$$

Where: $h(\mathrm{~A}, \Gamma \Rightarrow \Delta) \leq h(\Gamma \Rightarrow \Delta)$ and $h(\Gamma \Rightarrow \Delta, \mathrm{A}) \leq h(\Gamma \Rightarrow \Delta)$. The proof is omitted.

\subsubsection{Inversion rules}

$$
\begin{aligned}
& (\mathrm{I} \Rightarrow \rightarrow) \frac{\Gamma \Rightarrow \Delta, \mathrm{B} \rightarrow \mathrm{C}}{\mathrm{B}, \Gamma \Rightarrow \Delta, \mathrm{C}} \quad(\mathrm{I} \Rightarrow \underline{\forall}) \frac{\Gamma \Rightarrow \Delta, \forall x \mathrm{~B}[x]}{\mathrm{E} ! a, \Gamma \Rightarrow \Delta, \mathrm{B}[a]} \\
& (\mathrm{I} \Rightarrow \neg) \frac{\Gamma \Rightarrow \Delta, \neg \mathrm{A}}{\mathrm{A}, \Gamma \Rightarrow \Delta} \quad(\mathrm{I} \neg \Rightarrow) \frac{\neg \mathrm{A}, \Gamma \Rightarrow \Delta}{\Gamma \Rightarrow \Delta, \mathrm{A}}
\end{aligned}
$$




$$
(\mathrm{I} \rightarrow \Rightarrow 1) \frac{\mathrm{B} \rightarrow \mathrm{C}, \Gamma \Rightarrow \Delta}{\mathrm{C}, \Gamma \Rightarrow \Delta} \quad(\mathrm{I} \rightarrow \Rightarrow 2) \frac{\mathrm{B} \rightarrow \mathrm{C}, \Gamma \Rightarrow \Delta}{\Gamma \Rightarrow \Delta, \mathrm{B}}
$$

Where: the height $h$ of the conclusions is less than or equal to the height $h$ of the premises.

We want to state here just the proof of $(\mathrm{I} \Rightarrow \forall)$ :

There is a derivation of $\Gamma \Rightarrow \Delta, \forall x \mathrm{~B}[x]$ in $\mathbf{N}$ in tree-form. We keep track of the formula $\forall x \mathrm{~B}[x]$ beginning at the bottom of the tree. We replace each occurrence of $\forall x \mathrm{~B}[x]$ in the derivation with a formula $\mathrm{B}[a]$ and put E! $a$ left of $\Rightarrow$. If the branch originally ends with an axiom then after the application of this procedure the corresponding branch ends again with an axiom. If there is a $(\Rightarrow \forall)$-inference such that $a$ is subject to the restriction of variables, then we replace $a$ in this inference by a new variable (according to (Sub)). The number of logical inferences does not increase.

\subsubsection{Contraction}

$$
(\mathrm{C} \Rightarrow) \frac{\mathrm{A}, \mathrm{A}, \Gamma \Rightarrow \Delta}{\mathrm{A}, \Gamma \Rightarrow \Delta} \quad(\Rightarrow \mathrm{C}) \frac{\Gamma \Rightarrow \Delta, \mathrm{A}, \mathrm{A}}{\Gamma \Rightarrow \Delta, \mathrm{A}}
$$

Where: $h(\mathrm{~A}, \Gamma \Rightarrow \Delta) \leq h(\mathrm{~A}, \mathrm{~A}, \Gamma \Rightarrow \Delta)$ and $h(\Gamma \Rightarrow \Delta, \mathrm{A}) \leq h(\Gamma \Rightarrow \Delta, \mathrm{A}, \mathrm{A})$.

The proof is accomplished by induction on the complexity of $A$. Note that we us $(\forall \Rightarrow)$ to prove Contraction and that $(\forall \Rightarrow)$ is stated in such a way that this is possible.

\subsection{Admissibility of Cut}

In this section we want to prove in some detail that Cut is an indirectly admissible rule in $\mathbf{N}$. This result will be established in several steps.

\section{Cut}

$$
\frac{\Gamma \Rightarrow \Delta, \mathrm{A} \quad \mathrm{A}, \Gamma \Rightarrow \Delta}{\Gamma \Rightarrow \Delta}
$$

The admissibility of Cut is proved by double induction. The main induction is on the complexity of (the cut-formula) A and the auxiliary induction is on the height $h$ of the derivation of the right premise of the Cut.

We prove (Cut) in the above form and not in the version of (Cut*)

$$
\underline{\Gamma \Rightarrow \Delta, \mathrm{A} \quad \mathrm{A}, \Phi \Rightarrow \Pi}
$$




$$
\Gamma, \Phi \Rightarrow \Delta, \Pi
$$

However, as it is easily seen by the axioms and rules of $\mathbf{N}(\mathrm{Cut})$ and (Cut*) are derivable from each other.

The admissibility of Cut will be proved in several stages. We shall first define a formal system, called $\mathbf{N}^{\prime}$, as follows:

Let $\mathbf{N}^{\prime}$ be the formal system containing the axiom (Ax1) and the rules $(\Rightarrow P)$, $(\mathrm{P} \Rightarrow),(\neg \Rightarrow),(\Rightarrow \neg),(\rightarrow \Rightarrow),(\Rightarrow \rightarrow),(\forall \Rightarrow)$ and $(\Rightarrow \forall)$.

\subsubsection{Theorem.}

Cut is indirectly admissible in $\mathbf{N}^{\prime}$.

Proof.

We outline a few steps of the proof.

Basis

$\mathrm{A}$ is prime; i.e. $\mathrm{A}$ is of the form $\mathrm{E} ! t$ or $F^{n} t_{1} \ldots t_{\mathrm{n}}$.

There is a treelike derivation in $\mathbf{N}^{\prime}$ of $\Gamma \Rightarrow \Delta$, A and $A, \Gamma \Rightarrow \Delta$. We keep track of the formula $\mathrm{A}$ in $\Gamma \Rightarrow \Delta, \mathrm{A}$ - beginning at the bottom - and delete simultaneously every occurrence of $\mathrm{A}$. We delete the sequence $\mathrm{A}, \Gamma \Rightarrow \Delta$ and the whole right branch and obtain thereby a derivation of $\Gamma \Rightarrow \Delta$ in $\mathbf{N}^{\prime}$. By this method an axiom will be transformed into in axiom. One problem may occur: When there has been a basic sequent, i.e. an instance of an (Ax1)-axiom, at the top of a derivation branch, but now there is a sequent of the form $\Gamma \Rightarrow \Delta$; in this case we obtain a derivation of $\Gamma \Rightarrow \Delta$ from $\mathrm{A}, \Gamma \Rightarrow \Delta$ by putting this sequent and its derivation on top of $\Gamma \Rightarrow \Delta$ and then eliminating occurrences of $A$.

\section{Step}

$\mathrm{A}$ is $\neg \mathrm{B}$.

There is a treelike derivation in $\mathbf{N}^{\prime}$ of $\Gamma \Rightarrow \Delta, \neg \mathrm{B}$ and $\neg \mathrm{B}, \Gamma \Rightarrow \Delta$. Due to the inversion rules $(\mathrm{I} \Rightarrow \neg)$ and $(\mathrm{I} \neg \Rightarrow)$ we obtain derivations of $\mathrm{B}, \Gamma \Rightarrow \Delta$ and $\Gamma \Rightarrow \Delta$, B. $g(\mathrm{~B})<g(\neg \mathrm{B})$. Hence we may apply the inductive assumption and obtain: $\Gamma \Rightarrow$ $\Delta$.

In the case where $\mathrm{A}$ is of the form $\mathrm{B} \rightarrow \mathrm{C}$ we argue similarly.

$\mathrm{A}$ is $\forall x \mathrm{~B}[x]$.

This induction step is proved by induction on the minimal number of logical inferences, which are needed in order to obtain $\forall x \mathrm{~B}[x], \Gamma \Rightarrow \Delta$. The hypothesis of 
the main induction remains unchanged; i.e. the inductive assumption can be applied on formulas of a smaller degree than the original formula.

Basis of the side induction

If $h(\forall x \mathrm{~B}[x], \Gamma \Rightarrow \Delta)=0$, then $\forall x \mathrm{~B}[x], \Gamma \Rightarrow \Delta$ is an axiom. The conclusion $\Gamma \Rightarrow \Delta$ is derivable without logical inferences.

Side induction hypothesis

If $h(\forall x \mathrm{~B}[x], \Gamma \Rightarrow \Delta) \leq h(\Gamma \Rightarrow \Delta, \forall x \mathrm{~B}[x])$, then Cut is applicable with the cutformula $\forall x \mathrm{~B}[x]$ and the second premise $\forall x \mathrm{~B}[x], \Gamma \Rightarrow \Delta$. Note: We refer to premises of the last logical inference in a minimal derivation of $\forall x \mathrm{~B}[x], \Gamma \Rightarrow \Delta$.

We outline two cases: $(\neg \Rightarrow)$ - and $(\forall \Rightarrow)$-inferences.

The last logical inference is of the form: $(\neg \Rightarrow)$ :

$\underline{\forall \mathrm{B}}[\mathrm{x}], \Gamma \Rightarrow \Delta, \mathrm{C}(\neg \Rightarrow)$

$\neg \mathrm{C}, \forall x \mathrm{~B}[x], \Gamma \Rightarrow \Delta$

Thus $h(\forall x \mathrm{~B}[x], \Gamma \Rightarrow \Delta, \mathrm{C}) \leq h(\neg \mathrm{C}, \forall x \mathrm{~B}[x], \Gamma \Rightarrow \Delta)$. So Cut is applicable on $\Gamma$ $\Rightarrow \Delta, \forall x \mathrm{~B}[x]$ and $\forall x \mathrm{~B}[x], \Gamma \Rightarrow \Delta, \mathrm{C}$.

The cut-formula is $\forall x \mathrm{~B}[x]$ and the last logical inference in a minimal derivation of $\forall x \mathrm{~B}[x], \Gamma \Rightarrow \Delta$ is a $(\forall \Rightarrow)$-inference of the following form:

$\forall x \mathrm{~B}[x], \Gamma \Rightarrow \Delta, \mathrm{E} ! a \quad \mathrm{~B}[a], \forall x \mathrm{~B}[x], \Gamma \Rightarrow \Delta$

$\forall x \mathrm{~B}[x], \Gamma \Rightarrow \Delta$

The following holds: $h(\forall x \mathrm{~B}[x], \Gamma \Rightarrow \Delta, \mathrm{E} ! a) \leq h(\forall x \mathrm{~B}[x], \Gamma \Rightarrow \Delta)$ and $h(\mathrm{~B}[a]$, $\forall x \mathrm{~B}[x], \Gamma \Rightarrow \Delta) \leq h(\forall x \mathrm{~B}[x], \Gamma \Rightarrow \Delta)$. Hence, the hypothesis of the auxiliary induction is satisfied and the inductive assumption is applicable on $\Gamma \Rightarrow \Delta, \forall x \mathrm{~B}[x]$ and $\forall x \mathrm{~B}[x], \Gamma \Rightarrow \Delta, \mathrm{E} ! a$; result (1) $\Gamma \Rightarrow \Delta, \mathrm{E}$ !a. the inductive assumption is also applicable on $\Gamma \Rightarrow \Delta, \forall x \mathrm{~B}[x]$ and $\forall x \mathrm{~B}[x], \mathrm{B}[a], \Gamma \Rightarrow \Delta$; result: (2) $\mathrm{B}[a], \mathrm{E} ! a, \Gamma \Rightarrow$ $\Delta$. From $\Gamma \Rightarrow \Delta, \forall x \mathrm{~B}[x]$ we obtain by an application of $(\mathrm{I} \Rightarrow \forall)$ : (3) $\mathrm{E} ! a, \Gamma \Rightarrow \Delta$, $\mathrm{B}[a]$. Since the hypothesis of the main induction is satisfied we may apply successively the inductive assumption and obtain a derivation of $\Gamma \Rightarrow \Delta$.

This establishes the theorem.

\subsubsection{Intermediate Steps}

We call the system, that originates from $\mathbf{N}^{\prime}$ by adding of the rule Cut: $\mathbf{N}^{\prime} \mathbf{C}$. 
In order to prove the admissibility of Cut we follow a method proposed by Takeuti (1987, 34ff.).

We define a strings of formulas $\Phi_{\mathbf{E}}$ as follows: $\Phi_{\mathbf{E}}$ is the set of all formulas of the form $\mathrm{E} ! t \rightarrow t=t, s=t \rightarrow(\mathbf{D}[s] \rightarrow \mathbf{D}[t])$ and $\mathbf{D}[t] \rightarrow \mathrm{E} ! t$, which are constructible from the predicates and singular terms in $\Phi$.

If $\Gamma$ and $\Delta$ are strings of formula, then we write $(\Gamma \cup \Delta)$ for the string $\Gamma, \Delta .^{4}$

$\mathbf{N}$ plus Cut is called: $\mathbf{N C}$.

\subsubsection{Theorem (Hauptsatz).}

Cut is indirectly admissible in $\mathbf{N}$.

In order to prove that Cut is indirectly admissible in $\mathbf{N}$, we want to prove the following:

(S) If $\mathbf{N C} \vdash \Gamma \Rightarrow \Delta$, then $\mathbf{N} \vdash \Gamma \Rightarrow \Delta$.

(S) follows from:

(I) If $\mathbf{N C} \vdash \Gamma \Rightarrow \Delta$, then $\mathbf{N}^{\prime} \mathbf{C} \vdash \Gamma,(\Gamma \cup \Delta)_{\mathbf{E}} \Rightarrow \Delta$.

(II) If $\mathbf{N}^{\prime} \mathbf{C} \vdash \Gamma,(\Gamma \cup \Delta)_{\mathbf{E}} \Rightarrow \Delta$, then $\mathbf{N}^{\prime} \vdash \Gamma,(\Gamma \cup \Delta)_{\mathbf{E}} \Rightarrow \Delta$.

(III) If $\mathbf{N}^{\prime} \vdash \Gamma,(\Gamma \cup \Delta)_{\mathbf{E}} \Rightarrow \Delta$, then $\mathbf{N} \vdash \Gamma \Rightarrow \Delta$.

\section{Proof of (I)}

Basis: $h(\Gamma \Rightarrow \Delta)=0$.

There are four cases to consider.

Case 1

$\Gamma \Rightarrow \Delta$ is an axiom of the form $(\mathrm{Ax} 1)$, hence it also an axiom of $\mathbf{N}^{\prime} \mathbf{C}$. Case 2

$\Gamma \Rightarrow \Delta$ is an axiom of the form $(\mathrm{Ax} 2)$. E! $t \rightarrow t=t$ is a member of $(\Gamma \cup \Delta)_{\mathbf{E}}$. We have to prove that $\mathrm{E} ! t, \Gamma^{\prime}, \mathrm{E} ! t \rightarrow t=t, \Rightarrow \Delta, t=t$ is provable in $\mathbf{N}^{\prime} \mathbf{C}$.

$$
\begin{aligned}
& \mathrm{E} ! t, \Gamma \Rightarrow \Delta, t=t, \mathrm{E} ! t \quad t=t, \mathrm{E} ! t, \Gamma \Rightarrow \Delta, t=t
\end{aligned}
$$

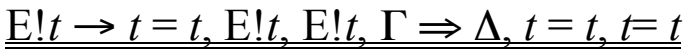

$$
\begin{aligned}
& \mathrm{E} ! t, \Gamma, \mathrm{E} ! t \rightarrow t=t, \Rightarrow \Delta, t=t
\end{aligned}
$$

Case 3

$\Gamma \Rightarrow \Delta$ is an axiom of the form (Ax3); $s=t \rightarrow(\mathbf{D}[s] \rightarrow \mathbf{D}[t])$ is a member of ( $\Gamma \cup$ $\Delta)_{\mathbf{E}}$. We have to show that $s=t, \mathbf{D}[s], \Gamma^{\prime}, s=t \rightarrow(\mathbf{D}[s] \rightarrow \mathbf{D}[t]) \Rightarrow \Delta^{\prime}, \mathbf{D}[t]$ is derivable in NC. The following is a derivation of that sequent:

\footnotetext{
${ }^{4}$ Here and below we make use of the notations ' $(\Gamma \cup \Delta)$ ' and ' $(\Gamma \cup \Delta)_{\mathbf{E}}$ '; these notations should not be understood as sets but as strings of formulas. This understanding is justified by the strucutural rules of $\mathbf{N}$.
} 


\section{Case 4}

$$
\begin{aligned}
& \underline{\mathbf{D}[s], \Gamma^{\prime} \Rightarrow \Delta^{\prime}, \mathbf{D}[s] \quad \mathbf{D}[t], \Gamma^{\prime} \Rightarrow \Delta^{\prime}, \mathbf{D}[t]}
\end{aligned}
$$

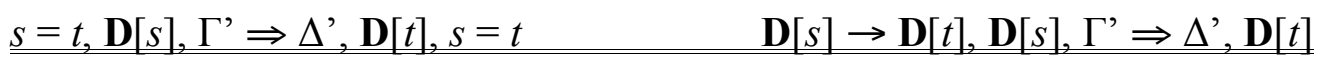

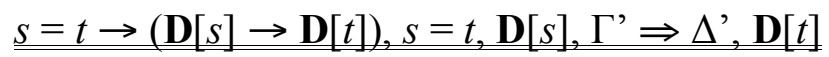

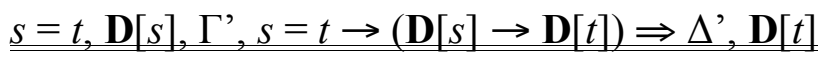

$$
\begin{aligned}
& \Gamma,(\Gamma \cup \Delta)_{\mathbf{E}} \Rightarrow \Delta
\end{aligned}
$$

$\Gamma \Rightarrow \Delta$ is an axiom of the form $(\mathrm{Ax} 4) ; \mathbf{D}[t] \rightarrow \mathrm{E}$ ! $t$ is a member of $(\Gamma \cup \Delta)_{\mathbf{E}}$. We have to prove that $\mathbf{D}[t], \Gamma^{\prime}, \mathbf{D}[t] \rightarrow \mathrm{E} ! t \Rightarrow \Delta^{\prime}, \mathrm{E} ! t$ is provable in $\mathbf{N C}$.

$$
\begin{gathered}
\frac{\mathrm{E} ! t, \Gamma \Rightarrow \Delta, \mathrm{E} ! t \quad \mathbf{D}[t], \Gamma \Rightarrow \Delta, \mathbf{D}[t]}{\mathbf{D}[t] \rightarrow \mathrm{E} ! t, \mathbf{D}[t], \Gamma \Rightarrow \Delta, \mathrm{E} ! t} \\
\mathbf{\mathbf { D } [ t ] , \Gamma { } ^ { \prime } , \mathbf { D } [ t ] \rightarrow \mathrm { E } ! t \Rightarrow \Delta , \mathrm { E } ! t}
\end{gathered}
$$

For the induction step, we have to argue that if the premise(s) of an inference rule is (are) derivable, then so is the conclusion. This part is easy and is therefore omitted.

The admissibility of Cut is already established for $\mathbf{N}^{\prime}$; therefore step (II) is already proved.

Proof of (III)

Basis: $h\left(\Gamma,(\Gamma \cup \Delta)_{\mathbf{E}} \Rightarrow \Delta\right)=0$.

There are again four cases to consider.

\section{Case 1}

If $\Gamma,(\Gamma \cup \Delta)_{\mathbf{E}} \Rightarrow \Delta$ is an axiom of the form $(A x 1)$, then it is also an axiom in $\mathbf{N}$.

\section{Case 2}

By assumption $\mathbf{N}^{\prime} \vdash \Gamma,(\Gamma \cup \Delta)_{\mathbf{E}} \Rightarrow \Delta$ s.t. $(\Gamma \cup \Delta)_{\mathbf{E}} \supseteq\{\mathrm{E} ! t \rightarrow t=t\} . \mathrm{E} ! t, \Gamma \Rightarrow \Delta, t$ $=t$ is an axiom of $\mathbf{N}$. We obtain from $\mathrm{E} ! t \rightarrow t=t, \Gamma \Rightarrow \Delta$ by means of the inversion rules $(\mathrm{I} \rightarrow \Rightarrow 1)$ and $(\mathrm{I} \rightarrow \Rightarrow 2)$ the following sequents:

(1) $t=t, \Gamma \Rightarrow \Delta$

(2) $\Gamma \Rightarrow \Delta, E ! t$

$\mathrm{E} ! t, \Gamma \Rightarrow \Delta, t=t$ is an axiom of $\mathbf{N}$. We may apply Cut and I-Cut to obtain a derivation of $\Gamma \Rightarrow \Delta$ in $\mathbf{N}$.

Case 3

By assumption $\mathbf{N}^{\prime} \vdash \Gamma,(\Gamma \cup \Delta)_{\mathbf{E}} \Rightarrow \Delta$, such that $(\Gamma \cup \Delta)_{\mathbf{E}} \supseteq\{s=t \rightarrow(\mathbf{D}[s] \rightarrow$ $\mathbf{D}[t])\}$. We obtain from this the sequent: 
(1) $s=t \rightarrow(\mathbf{D}[s] \rightarrow \mathbf{D}[t]), \Gamma \Rightarrow \Delta$

From (1) we obtain with an application of $(\mathrm{I} \rightarrow \Rightarrow 2)$ :

(2) $\Gamma \Rightarrow \Delta, s=t$

And again from (1) we obtain with an application of $(\mathrm{I} \rightarrow \Rightarrow 1)$ :

(3) $\mathbf{D}[s] \rightarrow \mathbf{D}[t], \Gamma \Rightarrow \Delta$

From (3) we get after applications of $(I \rightarrow \Rightarrow 2)$ and $(I \rightarrow \Rightarrow 1)$ the following sequents:

(4) $\Gamma \Rightarrow \Delta, \mathbf{D}[s]$

(5) $\mathbf{D}[t], \Gamma \Rightarrow \Delta$

The sequent

(6) $s=t, \mathbf{D}[s], \Gamma \Rightarrow \Delta, \mathbf{D}[t]$

is an axiom of $\mathbf{N}$. With an application of I-Cut on (6) and (2) we obtain

(7) $\mathbf{D}[s], \Gamma \Rightarrow \Delta, \mathbf{D}[t]$

From (7) and (4) and (5) with two applications of Cut we finally get $\Gamma \Rightarrow \Delta$.

\section{Case 4}

By assumption $\mathbf{N}^{\prime} \vdash \Gamma,(\Gamma \cup \Delta)_{\mathbf{E}} \Rightarrow \Delta$, such that $(\Gamma \cup \Delta)_{\mathbf{E}} \supseteq\{\mathbf{D}[t] \rightarrow$ E! $t\}$. By means of the inversion rules $(I \rightarrow \Rightarrow 2)$ and $(I \rightarrow \Rightarrow 2)$ we obtain the sequents:

(1) $\Gamma \Rightarrow \Delta, \mathbf{D}[t]$

(2) $\mathrm{E} ! t, \Gamma \Rightarrow \Delta$

$\mathbf{D}[t], \Gamma \Rightarrow \Delta, \mathrm{E} ! t$ is an axiom of $\mathbf{N}$; thus, we apply Cut twice and obtain a derivation of $\Gamma \Rightarrow \Delta$.

The induction step is analogous to the induction step for the proof of (I) and is omitted here.

This establishes the Hauptsatz for $\mathbf{N}$.

\subsection{Some Consequences of the Hauptsatz}

\subsubsection{Restricted Sub-formula Property}

\section{Definition}

(SF1) A is prime: A is a sub-formula of A.

(SF2) If $A$ is $\neg B$, then $\neg B$ and all subformulas of $B$ are sub-formulas of $A$.

(SF3) If $A$ is $B \rightarrow C$, then $B, C$ and all subformulas of $B$ and $C$ are sub-formulas of A.

(SF4) If $\mathrm{A}$ is $\forall x \mathrm{~B}[x]$, then $\mathrm{B}[t]$ for all $t$ are sub-formulas of $\mathrm{A}$.

Sub-formulas of a sequent $S$ are all sub-formulas of formulas of $S$. 
1.4.1.1 Theorem: Restricted Sub-formula Property.

If there is a tree-like derivation of $\Gamma \Rightarrow \Delta$ in $\mathbf{N}$, then all formulas occurring in $\Gamma \Rightarrow$ $\Delta$ are sub-formulas of this sequent - with the only exception of formulas of the form $\mathrm{E} ! t$ and $s=t$.

The proof is established by the length of a proof in $\mathbf{N}$.

\subsubsection{Corollary: Separation Property}

Any provable sequent $\Gamma \Rightarrow \Delta$ in $\mathbf{N}$ always has a derivation using only the logical rules and/or axioms for the logical operators occurring in $\Gamma \Rightarrow \Delta$. $^{5}$

Proof.

This corollary follows immediately from the theorem concerning the sub-formula property.

\subsubsection{Consistency}

Definition.

$\mathbf{N}$ is consistent iff $\ldots \Rightarrow \ldots$ (the empty sequent) is not derivable in $\mathbf{N}$.

\subsubsection{Theorem: $\mathbf{N}$ is consistent.}

\section{Proof.}

Suppose $\ldots \Rightarrow \ldots$ is derivable in $\mathbf{N}$, then there is a derivation in tree-form of $\ldots \Rightarrow \ldots$ in $\mathbf{N}$ without an application of Cut. Since the theorem concerning the subformula property holds, this is impossible.

\footnotetext{
${ }^{5}$ Cf. Troelstra/Schwichtenberg $(2000,106)$.
} 


\section{SEMANTICS}

There is a standard semantical approach to negative free logic (Morscher/Simons 2001, Lambert 2001, Burge 1991) and we shall follow it here. This approach consits basically of a partial interpretation-function $\varphi$ concerning singular terms of $L$; however, the extension of $\varphi$ to formulas and sequents of $L$ is total, i.e. those are either true or false under a given interpretation. It furthermore captures the underlying view from a standpoint of negative free logic, that every simple statement containing an empty singular term is false.

\subsection{Partial Interpration}

We define a partial interpretation $\mathfrak{\Im}=\langle D, \varphi\rangle$ as follows:

(1) $D$ is (a possibly empty) set,

(2) For every $t$, if $t$ is in the domain of $\varphi$, then $\varphi(t) \in D$, wehere $t$ is a FV.

(3) For every $n$-ary predicate $F^{n}: \varphi\left(F^{n}\right) \subseteq D^{n}$.

(4) There is a name $\underline{d}$ in $L$ (thereby $L$ is extended by those new individual free individual variables) for each object $d$ in $D$

The function $\varphi$ will be recursively extended as follows:

(5) $\varphi\left(F^{n} t_{1} \ldots t_{\mathrm{n}}\right)=\boldsymbol{t}$ iff $t_{i}(1 \leq i \leq n)$ is in the domain of $\varphi$ and $\left\langle\varphi\left(t_{1}\right), \ldots, \varphi\left(t_{\mathrm{n}}\right)\right\rangle$ $\in \varphi\left(F^{n}\right)$

(6) $\varphi(\mathrm{E} ! t)=\boldsymbol{t}$ iff $t$ is in the domain of $\varphi$.

(7) $\varphi(s=t)=t$ iff both $s$ and $t$ are in the domain of $\varphi$ and $\varphi(s)=\varphi(t)$.

(8) $\varphi(\neg \mathrm{A})=\boldsymbol{t}$ iff $\varphi(\mathrm{A})=\boldsymbol{f}$.

(9) $\varphi(\mathrm{A} \rightarrow \mathrm{B})=\boldsymbol{t}$ iff $\varphi(\mathrm{A})=\boldsymbol{f}$ or $\varphi(\mathrm{B})=\boldsymbol{t}$.

(10) $\varphi(\forall x \mathrm{~A}[x])=\boldsymbol{t}$ iff $\varphi(\mathrm{A}[\underline{d}])=\boldsymbol{t}$ for every object name $\underline{d}$.

(11) $\varphi(\Gamma \Rightarrow \Delta)=\boldsymbol{t}$ iff there is at least one A of $\Gamma$ such that $\varphi(\mathrm{A})=\boldsymbol{f}$ or there is at least one B of $\Delta$ such that $\varphi(\mathrm{B})=\boldsymbol{t}$.

The semantics developed meets the requirement stated for negative free logic, that every simple formula containing a non-denoting singular term is false.

\section{Definitions.}

A formula $\mathrm{A}$ is valid in a partial interpretation iff $\mathrm{A}$ is true in the partial interpretation (i.e. iff $\varphi(A)=1$ ).

A formula $\mathrm{A}$ is valid iff $\mathrm{A}$ is valid in all partial interpretations.

A set of formulas $\Gamma$ is valid in a partial interpretation iff every formula of $\Gamma$ is valid in this partial interpretation.

$\Gamma$ logically implies A iff A is valid in every interpretation in which $\Gamma$ is valid. 
$\Gamma \Rightarrow \Delta$ is valid iff $\Gamma \Rightarrow \Delta$ is true in every partial interpretation

$\Phi$ logically implies $\Gamma \Rightarrow \Delta$ iff $\Gamma \Rightarrow \Delta$ is valid in every interpretation in which $\Phi$ is valid.

\subsection{Soundness}

\section{Soundness of $\mathbf{N}$}

Every derivable sequent $\Gamma \Rightarrow \Delta$ in $\mathbf{N}$ is valid.

The proof is as usual.

\subsection{Completeness and Compactness}

In this section we will prove the completeness and (countable) compactness theorem for $\mathbf{N}$. We employ the methods of S. Buss $(1998,34 \mathrm{ff})$ in order to establish the theorems.

\section{Theorems.}

Let $\Gamma \Rightarrow \Delta$ be a sequent of the language $\mathbf{L}$.

(1) If $\Gamma \Rightarrow \Delta$ is valid, then it has a cut-free proof.

(2) If $\Phi$ be a set of formulas. If $\Phi$ logically implies $\Gamma \Rightarrow \Delta$, then there are $\mathrm{C}_{1}, \ldots, \mathrm{C}_{k} \in \Phi$ s.t. $\mathrm{C}_{1}, \ldots, \mathrm{C}_{k}, \Gamma \Rightarrow \Delta$ has a cut-free proof.

We mean by a "cut-free proof" a proof without any application of Cut, but there may be some applications of I-Cut.

(1) states the completeness theorem and (2) the compactness theorem. Like in classical logic (2) implies (1). So we outline here the proof of (2).

The main idea of the proof is as follows: try to construct a derivation of $\Gamma \Rightarrow \Delta$ in $\mathbf{N}$ from the bottom up to the initial sequents. ${ }^{6}$ Because of the quantifier $\forall$ the proof-search procedure can be infinite. However, if the proof-search procedure does not terminate, then the sequent $\Gamma \Rightarrow \Delta$ is not valid (cf. Buss (1998, 34) and there is a "counter"-intpretation based on this proof-search procedure.

The language $\mathbf{L}$ is countable, thus we may enumerate all $\mathbf{L}$-formulas as $A_{1}$, $\mathrm{A}_{2}, \mathrm{~A}_{3}, \ldots$ and we may enumerate all $\mathbf{L}$-terms as $t_{1}, t_{2}, t_{3}, \ldots$ in such a way that every formula and every term occurs infinitely often in this enumeration.

We shall attempt to construct a (cut-free) proof $\mathbf{P}$ of $\Gamma \Rightarrow \Delta$. The construction of $\mathbf{P}$ proceeds in stages.

\footnotetext{
${ }^{6}$ Kleene (1967) presents a very good exposition of this kind of completeness proofs.
} 
Initially, $\mathbf{P}$ consists of just the sequent $\Gamma \Rightarrow \Delta$. At each stage, $\mathbf{P}$ will be modified. A sequent in $\mathbf{P}$ is said to active provided that it is a leaf sequent, and it is not an axiom.

Each stage of the construction of $\mathbf{P}$ considers a pair $\left\langle\mathrm{A}_{i}, t_{j}\right\rangle$.

At each state we do the following:

Loop: Let $\angle \mathrm{A}_{i}, \mathrm{t}_{j}>$ be the next pair in the enumeration.

Step (1): If $\mathrm{A}_{i}$ is in $\Phi$, then replace every sequent $\Gamma^{\prime} \Rightarrow \Delta^{\prime}$ in $\mathbf{P}$ with the sequent $\Gamma^{\prime}, \mathrm{A}_{i} \Rightarrow \Delta^{\prime}$.

Step (2): If $\mathrm{A}_{i}$ is prime, do nothing and proceed to the next stage. Otherwise, we will modify $\mathbf{P}$ at the active sequents, which contain $\mathrm{A}_{i}$, by doing one of the following:

Case (2a): If $\mathrm{A}_{i}$ is $\neg \mathrm{B}$, then every active sequent in $\mathbf{P}$, which contains $\mathrm{A}_{i}$ of the form $\neg \mathrm{B}$ is replaced by

$\neg \mathrm{B}, \Gamma^{\prime} \Rightarrow \Delta^{\prime}, \mathrm{B}$

$\neg \mathrm{B}, \Gamma^{\prime} \Rightarrow \Delta^{\prime}$

Every active sequent in $\mathbf{P}$ of the form $\Gamma^{\prime} \Rightarrow \Delta^{\prime}, \neg \mathrm{B}$ is replaced by

$\underline{\mathrm{B}, \Gamma^{\prime} \Rightarrow \Delta^{\prime} \neg \mathrm{B}}$

$\Gamma^{\prime} \Rightarrow \Delta^{\prime}, \neg \mathrm{B}$

Case (2b): If $\mathrm{A}_{i}$ is of the form $\mathrm{B} \rightarrow \mathrm{C}$, then every active sequent in $\mathbf{P}$ of the form $\mathrm{B} \rightarrow \mathrm{C}, \Gamma^{\prime} \Rightarrow \Delta^{\prime}$ is replaced by

$$
\frac{\mathrm{B} \rightarrow \mathrm{C}, \Gamma^{\prime} \Rightarrow \Delta^{\prime}, \mathrm{B} \quad \mathrm{C}, \mathrm{B} \rightarrow \mathrm{C}, \Gamma^{\prime} \Rightarrow \Delta^{\prime}}{\mathrm{B} \rightarrow \mathrm{C}, \Gamma^{\prime} \Rightarrow \Delta^{\prime}}
$$

Every active sequent in $\mathbf{P}$ of the form $\Gamma^{\prime} \Rightarrow \Delta^{\prime}, \mathrm{B} \rightarrow \mathrm{C}$ is replaced by the derivation

$$
\frac{\mathrm{B}, \Gamma^{\prime} \Rightarrow \Delta^{\prime}, \mathrm{B} \rightarrow \mathrm{C}, \mathrm{C}}{\Gamma^{\prime} \Rightarrow \Delta^{\prime}, \mathrm{B} \rightarrow \mathrm{C}}
$$

Case (2c): If $\mathrm{A}_{i}$ is of the form $\forall x \mathrm{~B}[x]$, then every active sequent in $\mathbf{P}$ of the form $\forall x \mathrm{~B}[x], \Gamma^{\prime} \Rightarrow \Delta^{\prime}$ is replaced by the derivation

$$
\frac{\mathrm{B}\left[t_{i}\right], \forall x \mathrm{~B}[x], \Gamma^{\prime} \Rightarrow \Delta^{\prime} \quad \forall x \mathrm{~B}[x], \Gamma^{\prime} \Rightarrow \Delta^{\prime} \mathrm{E} ! t_{i}}{\forall x \mathrm{~B}[x], \Gamma^{\prime} \Rightarrow \Delta^{\prime}}
$$

(Remember the enumeration of all pairs $<\mathrm{A}_{i}, t_{j}>$ )

Any active sequent of the form $\Gamma^{\prime} \Rightarrow \Delta^{\prime}, \forall x \mathrm{~B}[x]$ is replaced by the derivation $\mathrm{E} ! a, \Gamma^{\prime} \Rightarrow \Delta^{\prime}, \forall x \mathrm{~B}[x], \mathrm{B}[a]$ 
where $a$ is new in $\mathbf{P}$.

$$
\Gamma^{\prime} \Rightarrow \Delta^{\prime}, \forall x \mathrm{~B}[x]
$$

Step (3):

If there are no active sequents remaining in $\mathbf{P}$, exit from the loop; otherwise continue with the next loop iteration.

If the algorithm constructing $\mathbf{P}$ ever halts, then $\mathbf{P}$ gives a (cut-free) proof of $\mathrm{C}_{l}, \ldots$, $\mathrm{C}_{k}, \Gamma \Rightarrow \Delta$ for some $\mathrm{C}_{1}, \ldots, \mathrm{C}_{k} \in \Phi$.

It remains to show that if the construction of $\mathbf{P}$ never halts, then the sequent $\Gamma \Rightarrow \Delta$ is not logically implied by $\Phi$.

Suppose the above construction of $\mathbf{P}$ never halts and consider the result of applying the entire infinite construction process. $\mathbf{P}$ will be an infinite tree. $\mathbf{P}$ is a finitely branching tree, so by König's Lemma, there is at least one infinite branch $\pi$ in $\mathbf{P}$ starting at the roots and proceeding up through the tree. We use $\pi$ to construct a partial interpretation.

$D^{\prime}=\{t: t$ occurs at least once in a formula of the form E! $t$ in an antecedent of a sequent of $\pi\}$

We define a relation $\sim$ on $D$ as follows:

$$
s \sim t: \leftrightarrow s=t \text { occurs in } \pi \text { on the left side of a sequent }
$$

The relation $\sim$ is an equivalence relation on $D$ (i.e. on the set of existents), since the following sequents are provable in $\mathbf{N}$ :

(1) $\vdash \mathrm{E} ! t \Rightarrow t=t$

(2) $\vdash s=t \Rightarrow t=s$

(3) $\vdash s=r, r=t \Rightarrow s=t$

(4) $\vdash \mathrm{E} ! t, s=t \Rightarrow \mathrm{E} ! s$

Since $\mathbf{N} \vdash s=t, \mathrm{~A}[s] \Rightarrow \mathrm{A}[t]$ the relation $\sim$ is a congruence relation.

Next we define a partial interpretation $\varphi$ over $D$ as follows:

(1) If $t \in D$, then $\varphi(t)=\tilde{t}$.

(2) $\varphi\left(F^{n}\right)=\left\{\left\langle t_{1}^{\sim}, \ldots, t_{n}^{\sim}>: F^{n} t_{1} \ldots t_{n}\right.\right.$ occurs on the left of $\left.\pi\right\}$

It has to be proved by induction on the complexity of A, that every formula in the antecedent of $\pi$ is true in this partial structure and and every formula in the succedent of $\pi$ is false. We shall state here the "critical" case for $\mathrm{A}=\forall x \mathrm{~B}[x]$. We outline the case for $\mathrm{A}=\forall x \mathrm{~B}[x]$. 
A occurs in the antecedent. Due to the algorithm $\pi$ branches either trough (i) $\mathrm{B}[t]$, $\forall x \mathrm{~B}[x], \Gamma \Rightarrow \Delta$ or through (ii) $\forall x \mathrm{~B}[x], \Gamma \Rightarrow \Delta, \mathrm{E}$ ! t. Suppose (i): $\varphi(\mathrm{B}[t]=\boldsymbol{t}$ for every $t$, since with respect to the algorithm eventually every $t$ occurs in $\pi$; thus $\varphi(\mathrm{A})=\boldsymbol{t}$. Suppose (ii): $\varphi(\mathrm{E} ! t)=\boldsymbol{f}$, for every $t$, since eventually every $t$ will occur in $\pi$. With respect to the semantic clause for $\forall$-formulas and by (2) above: $\varphi(\mathrm{A})=\boldsymbol{t}$. If $\mathrm{A}$ occurs in the succedent of a sequent of $\pi$, then there is a free variable $a$ such that $\mathrm{E} ! a$ occurs in antecedent and $\mathrm{B}[a]$ occurs in a succedent of $\pi$. Hence $\varphi(\mathrm{E} ! a)=$ $\boldsymbol{t}$ and $\varphi(\mathrm{B}[a])=\boldsymbol{f}$, thus $\varphi(\mathrm{A})=\boldsymbol{f}$.

\section{Acknowledgement}

I am indepted to Johannes Czermak and anonymous referees for valuable comments on earlier drafts of this paper.

\section{References}

Aristotle (1984): The Complete Works of Aristotle. The Revised Oxford Translation, vol. 1, Jonathan Barnes (ed.), Princeton/NJ: Princeton University Press.

Burge, Tyler (1991): "Truth and Singular Terms", in: Lambert, K. (1991, ed.), 189-204.

Buss, S. (1998): “An Introduction to Proof Theory", in: Buss, S. (1998, ed.): Handbook of Proof Theory, Amsterdam-Lausanne-New York-Oxford: Elsevier, 1-78.

Gentzen, G. (1934/35): Untersuchungen über das logische Schliessen: Math. Zeitschrift 39, 1934-5, 176-210, 405-431.

Gumb, R. (2000): "Model Sets in a Nonconstructive Logic of Partial Terms with Definite Descriptions", in: Dyckhoff, R. (ed. 2000): Automated Reasoning with Analytic Tableaux and Related Methods, International Conference, TABLEAUX 2000, St. Andrews, Scotland, UK, July 2000, vol. 1847 of Lecture Notes in Artificial Intelligence, Berlin: Springer, 268-278.

Gumb, R.D. (2001): "An Extended Joint Consistency Theorem for a Nonconstructive Logic of Partial Terms with Definite Descriptions", in: Studia Logica 69, 279-292.

Hintikka, Jaakko (1964): "Definite descriptions and self-identity", in: Philosophical Studies, 1964, 5-7.

Kleene, S. C. (1967): Mathematical Logic, New York: John Wiley \& Sons.

Lambert, K. (2001): "Free Logics”, in: L. Goble (2001, ed.): The Blackwell Guide to Philosophical Logic, Malden, Ma.: Blackwell, 258-279. 
Lambert, K. (1997): Free Logics: Their Foundations, Character and Somme Applications Thereof, Sankt Augustin: Academia Verlag.

Lambert, K. (1991): Philosophical Applications of Free Logic, Oxford: Oxford University Press.

Morscher, E./Hieke, A. (2001, eds.): New Essays in Free Logic In Honour of Karel Lambert, Dordrecht-Boston-London: Kluwer.

Morscher, E./Simons, P.M. (2001): "Free Logic: A Fifty-Year Past and an Open Future", in: Morscher/Hieke (2001, eds.), 1-34.

Scales, Ronald D. (1969): Attribution and Existence, Ph.D. Thesis (University of California, Irvine).

Scott, Dana S. (1967): "Existence and Description in Formal Logic", in: Schoenmann, R. (1967, ed.): Bertrand Russell, Philosopher of the Century, London: Allen and Unwin: 181-200. (Reprinted in Lambert (1991, ed.))

Schock, Rolf (1968): Logics without Existence Assumptions, Stockholm: Almqvist \& Wiksell.

Takeuti, G. ( $\left.{ }^{2} 1987\right)$ : Proof Theory, Amsterdam-Lausanne-New York-OxfordTokyo: North Holland.

Troelstra, A. S./Schwichtenberg, H. (2000): Basic Proof Theory, Cambridge: Cambridge University Press. 